\title{
Situation afghanischer Geflüchteter
}

In diesem Kapitel sollen überblicksartig die wichtigsten Informationen zur Lage afghanischer Asylbewerber*innen zusammengefasst werden. Dieses Wissen ist entscheidend, um die empirischen Ergebnisse besser einordnen und verstehen zu können. Zunächst werden Zahlen und Fakten zur Lage Afghanistans geboten (Abschn. 3.1), um darauf aufbauend die rechtliche Situation afghanischer Asylbewerber*innen in Deutschland darzustellen (Abschn. 3.2). Der Fokus liegt auf Unterbringungs- (Abschn. 3.2.1) und Asylverfahrensgesetze (Abschn. 3.2.2). Abgeschlossen wird das Kapitel mit einer kurzen Reflexion über den Begriff „Flüchtlingskrise“ (Abschn. 3.3).

\subsection{Afghanistan als Herkunftsland}

Afghanistan, als eines der Hauptherkunftsländer von Geflüchteten liegt an einer geopolitischen Schnittstelle, an der Einflusssphären unterschiedlicher Staaten und machtpolitischer Sicherheitskomplexe zusammentreffen (Voje 2014: 293). Als Binnenland mit einer ungefähr doppelt so großen Fläche wie Deutschland ${ }^{1}$ und einer Bevölkerung von ca. 35,5 Millionen lässt sich Afghanistan nicht einem eindeutigen Kulturraum zuordnen (Schetter 2010: 18). Über die kulturelle und ethnische Vielfalt Afghanistans darf trotz des vorherrschenden Islam, dem 99 Prozent der Bevölkerung angehören, nicht hinweggesehen werden (ebd.: 19). In dem Land finden sich weit über 30 verschiedene Sprachen, von denen die wichtigsten Dari - die afghanische Ausführung von Farsi - und Paschtu sind (ebd.: 22).

\footnotetext{
${ }^{1}$ Afghanistan hat eine Fläche von $652.860 \mathrm{~km}^{2}$ (Statistisches Bundesamt 2018: 3).

(C) Der/die Autor(en) 2021

K. Behnam Shad, Die emotionale Erfahrung des Asyls, https://doi.org/10.1007/978-3-658-31308-1_3
} 
Usbekisch, Tadschikisch, Turkmenisch und Belutschisch sind weitere Hauptsprachen des Landes (ebd.). Die Sprachenvielfalt, insbesondere am Hindukusch, ist erwähnenswert, da sich die Sprachen und Ethnien in diesen Gebieten so stark vermischen, dass der Faktor Sprache nicht mehr sinnvoll als ethnisches Kriterium eingesetzt werden kann (ebd.). Ohnehin wäre eine Unterteilung der afghanischen Bevölkerung in deutlich abgegrenzte Ethnien nicht unbedenklich, da Identitäten sowie soziale und andere Zugehörigkeiten häufig vermischt sind. Von den über 50 Ethnien sind die größten Gruppen die Paschtunen - die bis auf kurze Phasen seit der Gründung 1747 die Führung des Landes stellen -, die Tadschiken, Usbeken, Belutschen, Nuristani und Hazara (Meienberg 2012: 66). Die Gruppe der Paschtunen, die sich in Durrani und Ghilzai unterteilen, ist mit ca. 26 Millionen die größte Stammesgesellschaft (ebd.: 65). Die schiitische Minderheit der Hazara aus dem zentralasiatischen Hazarajat ist die am stärksten benachteiligte und ausgegrenzte ethnische Gruppe Afghanistans (Schetter 2010: 26).

Die seit dem 11. September 2001 zunehmende geopolitische Bedeutung des Landes in Zentralasien für die Vereinigten Staaten von Amerika ist historisch betrachtet nichts Außergewöhnliches für Afghanistan. Zwischen dem persischen Plateau und dem Himalaya-Gebirge, an der Nahtstelle unterschiedlicher Großreiche, bildete das Land bereits zu vorchristlicher Zeit einen „highway of conquest“" (ebd.). Doch seit dem beginnenden 21. Jahrhundert avanciert Afghanistan in der öffentlichen Wahrnehmung immer mehr zum „Gegenpol der zivilisierten Welt" und zur "Schattenseite der Globalisierung" (Schetter 2010: 9). Hier zeigt sich die Kluft zwischen „Moderne“ und „Tradition“ vor allem in den gegensätzlichen Ansichten zu Demokratie und Menschenrechten. Während weite Teile der ländlichen Bevölkerung solche Prinzipien als unislamisch zurückweisen, beabsichtigt die urban geprägte Bevölkerung eine zügige Säkularisierung des Landes. $\mathrm{Zu}$ den erwähnten geopolitischen Interessen fügen sich zahllose Interessengruppen, vertreten durch die neu entstandenen Kriegsfürstentümer innerhalb des Landes, die ein staatliches Gewaltmonopol verhindern. Nichtwestliche Machtformierungen und die Einführung demokratischer Prinzipien werden die beschriebene Kluft angesichts des ungelösten Grundproblems der Organisation der zahlreichen mächtigen Stammesgruppierungen eher vergrößern als verringern (ebd.: 150). Afghanistan ist seit dem Einmarsch der Sowjetunion 1979 und dem darauffolgenden Krieg eines der Hauptherkunftsländer von Geflüchteten geworden (Luft 2016: 32). Mehr als die Hälfte der Bevölkerung verließ das Land, 4,5 Millionen Menschen flüchteten in die Nachbarstaaten Pakistan und Iran. Derzeit leben über 1 Millionen registrierte und etwa 1,5 bis 3 Millionen nicht registrierte Afghan*innen in der Islamischen Republik, die die größte Anzahl afghanischer Geflüchteter weltweit beherbergt (UNHCR 2019). Pakistan beherbergt rund 
2 Millionen Menschen aus Afghanistan, aus dem allein 2017 über 2,6 Millionen Menschen geflüchtet sind (UNO-Flüchtlingshilfe 2018). Die 1 Millionen Binnenvertriebenen sollten in diesem Zusammenhang ebenfalls berücksichtigt werden.

\subsubsection{Deutschlands Rolle in Afghanistan}

Im Auftrag der Vereinten Nationen leisteten die internationalen Truppen der International Security Assistance Force (ISAF) seit 2001 dreizehn Jahre lang ihren Beitrag, um die afghanische Regierung bei einer flächendeckenden Vormachtstellung mittels Sicherheitskräften und damit einem umfassenden Staatsaufbau zu unterstützen (Münch 2015: 1). Deutschland war von Anfang an beteiligt, wobei zunächst lediglich eine sechsmonatige Friedensmission beabsichtigt war (Wiegold 2016). Die Bundesrepublik stellte zu Beginn mit der Bundeswehr den drittgrößten Anteil an der internationalen Schutztruppe und darüber hinaus zwei ISAF-Kommandeure (Münch 2015: 2). Laut der international renommierten Initiative Campaign Against Arms Trade (CAAT) lieferte die Bundesrepublik zudem zwischen 2002 und 2015 Rüstungsartikel im Wert von über 365 Millionen Euro nach Afghanistan (CAAT 2019). Der deutsche Einsatz in Afghanistan begann als Friedenseinsatz mit geringem Kräfteeinsatz und entwickelte sich zu einem Aufstandsbekämpfungseinsatz, bei dem Gefechte und Anschläge zum Alltag gehören (Seiffert et al. 2012: 12). Zunächst sollte die Bundeswehr lediglich die afghanische Regierung beschützen. Dies änderte sich 2010 mit dem Mandat zum Schutz der Zivilbevölkerung (ebd.). Ein Großteil der afghanischen Zivilbevölkerung erhoffte sich mit der Friedensmission und dem 2005 auf den Weg gebrachten Entwicklungsstrategiepapier eine grundlegende Transformation zugunsten der Menschenrechte innerhalb Afghanistans (Kargar 2012: 327). Die Leitidee des Papiers war es, mehr Menschenrechte und soziale Gerechtigkeit durch staatliche Sicherheit zu gewährleisten (Islamic Republic of Afghanistan 2005: 17). Faktisch verschlechterte sich jedoch nach dem Beschluss des Entwicklungsstrategiepapiers die menschenrechtliche Situation landesweit (Niland 2004: 61). Im Jahr 2011 war Deutschland mit 5.334 Soldat*innen der Bundeswehr in Afghanistan vertreten (Bundeswehr 2019). 53 deutsche Soldat*innen starben bei Einsätzen in Afghanistan (Bundesregierung 2014: 36). Am 1. Januar 2015 folgte schließlich die NATO-geführte Resolute Support Mission (RSM), deren Auftrag die Ausbildung, Beratung und Unterstützung afghanischer Streitkräfte war. Gemäß dem Beschluss vom 21. März 2019 können fortan 1.300 deutsche Soldat*innen in Afghanistan eingesetzt werden (Bundeswehr 2019). 
Seit dem ISAF-Einsatz hat in Afghanistan die Korruption, besonders im entscheidenden Bereich der Justiz, zugenommen (Bundesregierung 2014: 11 f.), wie ebenfalls von der Nichtregierungsorganisation Integrity Watch Afghanistan in regelmäßig publizierten Berichten bestätigt wird (Integrity Watch Afghanistan 2014). Korruption bildet, gleich hinter Sicherheitsfragen, die größte Sorge der afghanischen Zivilbevölkerung (Bundesregierung 2014: 15). Zudem verringerten sich nicht die sogenannten „Sicherheitsvorfälle“ - gemeint sind militärische Angriffe aufständischer Gruppierungen gegen Regierungskräfte und internationale Truppen (Münch 2015: 2). Hinzu kommt der gesteigerte Drogenkonsum des ,weltgrößten Produzenten von Opium“ (Bundesregierung 2014: 13): Zwischen 2004 und 2009 stieg der Gebrauch von Heroin innerhalb der afghanischen Bevölkerung um $140 \%$ (UNODC 2014). Die festgefahrene Situation und die wenig optimistischen Berichte gaben den Ausschlag für die veränderte inhaltliche Dimension der Politik westlicher Entscheidungsträger*innen, die ihre Ansprüche im Bereich des Staatsaufbaus auf die Devise „Afghan Good Enough“ reduzierten (Münch 2015: 2). In Zukunft wird Deutschland überwiegend Soldat*innen im Norden Afghanistans sowie im Großraum Kabuls stationieren, was nach dem NATO-Afghanistan-Truppenstatusabkommen (NATO-Afgh.-SOFA) die Bundesrepublik zum zweitgrößten Truppensteller macht (Bundesregierung 2014: 6). Mit dem NATO-Afgh.-SOFA, das am 30. September 2014 in Kabul unterzeichnet wurde, eröffnete sich für die NATO die Möglichkeit, die afghanischen Sicherheitskräfte (ANSF) ab dem 1. Januar 2015 durch die RSM weiter zu unterstützen (Bundesregierung 2014: 5). Deutschland finanziert die ANSF mit jährlich 200 Millionen US-Dollar (ebd.: 21). Ebenso stellte die Bundesrepublik in der unter der afghanischen Unabhängigen Wahlkommission (IEC) durchgeführten Wahlüberprüfung - „Audit“ - mit 65 Wahlbeobachter*innen und über 470 Einsätzen nach den USA das umfassendste Beobachtungskontingent der IEC (ebd.: 10). Für die vorliegende Arbeit ist von Bedeutung, dass die Hauptakteur*innen des deutschen Engagements die Rüstungsindustrie sowie die Bundeswehr sind. Zwar hat Deutschland in militärischer Hinsicht deutlich weniger Gewicht als die USA, ist aber dennoch durchweg an Entscheidungen von elementarer Bedeutung für Afghanistan beteiligt, wie die seit 2001 unregelmäßig in Bonn abgehaltenen Afghanistankonferenzen ${ }^{2}$, welche die politischen, wirtschaftlichen und militärischen Aspekte des Landes koordinieren, zeigen (Münch 2015: 2).

\footnotetext{
${ }^{2}$ Auch als „Petersberg-Konferenz“ oder „Afghanistan-Compact“ bekannt.
} 
Es lässt sich festhalten, dass Deutschland von 2001 bis 2014 an einem Krieg beteiligt war, ohne die Afghanistanfrage fest und unverrückbar in die deutsche und somit europäische Politik zu integrieren, um sich nicht, wie etwa Großbritannien, von den Vereinigten Staaten von Amerika abhängig zu machen (Daxner 2015). Abgesehen von vielen überzogenen Zielsetzungen der Bundesrepublik - die Emanzipation von Frauen Afghanistans, die Reformierung der afghanischen Polizei oder die flächendeckende Stärkung der Menschenrechte, um einige zu nennen - ist die Bilanz der deutschen Intervention recht dürftig. Insbesondere hätte Deutschland mehr auf die Belange und Nöte der lokalen afghanischen Bevölkerung eingehen sollen. Die Afghanistanpolitik Deutschlands wird extern an das Land herangetragen (ebd.) und kann als eine Spielart (neo-)kolonialer Politik verstanden werden. Bei der Rechtfertigung von Auslandseinsätzen der deutschen Bundeswehr ist zudem eine wesentliche Akzentverschiebung festzustellen: Es wird immer selbstverständlicher, nicht vorrangig moralische Fragen, sondern wirtschaftspolitische Interessen als Argument anzuführen (Haid et al. 2011: 49). So stellt eine Studie des Zentrums für Transformation der Bundeswehr mit Blick auf die sicherheitspolitischen Herausforderungen durch knapper werdende Energieressourcen fest, dass die bereits existierende Selektivität militärischer Interventionen zunehmen wird:

\begin{abstract}
„Damit konzentriert sich das Interesse an (Entwicklungs-)Zusammenarbeit auf diejenigen Fälle, wo Entwicklungsländer mittel- und langfristig den Zugang zu wichtigen Ressourcen öffnen. Damit treten neben den unmittelbar Öl und Energierohstoffe exportierenden Nationen auch bestimmte Staaten im unmittelbaren Umfeld in den Vordergrund, die eine Bedeutung für den Transport dieser Güter besitzen. Das Verhalten von Staaten wird in diesem Sinne ,egoistischer" und noch stärker an den eigenen Interessen ausgerichtet - Idealismus tritt als Triebfeder für außenpolitisches Engagement deutlich in den Hintergrund." (Zentrum für Transformation der Bundeswehr 2010: 45)
\end{abstract}

Die zunehmende Militarisierung geht Hand in Hand mit einer Außenpolitik, die, ebenso wie die Entwicklungszusammenarbeit, Afghanistan lediglich von ,außen“ betrachtet und definiert (Daxner 2015). Dies hat erhebliche Konsequenzen und Implikationen für die Interventionen Deutschlands. Zudem werden in Afghanistan Entwicklungsgelder in engem Zusammenhang mit dem dort herrschenden militärischen Souverän eingesetzt (Haid et al. 2011: 39). Viele namhafte Hilfsorganisationen wie Caritas International oder Ärzte ohne Grenzen wandten sich bereits öffentlich gegen diese Instrumentalisierung von Hilfsgeldern (ebd.). Dass Organisationen wie Ärzte ohne Grenzen unter der sowjetischen Herrschaft und sogar während der Bürgerkriege unter den Taliban humanitäre Hilfe 
zu leisten imstande waren, aber am 28. Juli 2004 unter der zivil-militärischen Besatzung des Westens aufgrund der erzwungenen Unterordnung unter das Militär Afghanistan verlassen mussten (Haydt 2006: $320 \mathrm{f}$; Haid et al. 2011: 39 f.), ist eine bemerkenswerte Tatsache ${ }^{3}$. Angesichts der bewussten und höchstproblematischen Vermischung humanitärer Hilfe mit militärischen Zielen, die den eigentlichen Sinn Ersterer aushöhlt, hätte Deutschland in der Zeit ab 2004 eine eigene entwicklungspolitische Strategie auf den Weg bringen können immerhin ist die zivile Befriedung ausdrücklich das Anliegen der Mission. Mit der Verknüpfung von Militarisierung und Entwicklungszusammenarbeit verkennt die Außenpolitik Deutschlands den Stellenwert der indigenen Gesellschaften Afghanistans. In diesem Zusammenhang spricht die postkoloniale Theoretikerin Nikita Dhawan von der Gefahr der Missachtung indigener Gruppen (2009: 57). Die grundlegenden Pfeiler der guten Regierungsführung - Vertrauen, Partizipation und Selbstbestimmung - werden von außerhalb, also gewissermaßen „kolonisierend“, an das Land herangetragen und der afghanischen Bevölkerung übergestülpt (Daxner 2015). Das erwähnte Aufblühen in der Drogenökonomie und die mit ihr verbundene Korruption und Vetternwirtschaft heben die Plan- und Ratlosigkeit der intervenierenden Kräfte in diesem Krieg hervor. Deutschland reiht sich damit in eine Gruppe von ,mächtigen, westlichen Staaten [ein], die sich wenige Jahrzehnte zuvor reumütig vom Imperialismus verabschiedet haben“ (Stromseth et al. 2006: 2) und nun verstärkt im Namen der Menschenrechte und der Demokratie auf militärische Gewalt in den Gebieten anderer Länder zurückgreifen. Besonders in Afghanistan wird die militärische Dimension der Intervention deutlich (Haid et al. 2011: 39). Deutschland sollte eine klare Position zu der Frage finden, inwiefern Afghanistan ein Zielland deutscher Entwicklungszusammenarbeit darstellt, ohne sich wie bisher von geostrategischen Interessen der Vereinigten Staaten, Chinas und Russlands instrumentalisieren zu lassen (Daxner 2015). Doch dies scheint knapp zwei Jahrzehnte nach Beginn des Einsatzes äußerst schwierig zu sein, vor allem angesichts der Fragilität des Landes am Hindukusch, das nunmehr vom Islamischen Staat (IS) vereinnahmt wird (UNHCR 2016a: 3).

\footnotetext{
${ }^{3} \ddot{A} r z$ te ohne Grenzen konnten ihr Engagement in Afghanistan 2009 wieder aufnehmen.
} 


\subsubsection{Aktuelle Lage}

Gemäß dem UN-Sicherheitsbericht von 2017 ist die Zahl der sicherheitsrelevanten Zwischenfälle gestiegen (UN General A.S.C. 2017). Der innerstaatliche Konflikt hat sich im gesamten Land ausgebreitet (Mesovic/Pichl 2016: 10). Besonders besorgniserregend ist hierbei die Veränderung der Strategie der Taliban vom herkömmlichen Guerillakrieg hin zu groß angelegten Angriffen in städtischen Gebieten (UNHCR 2016a: 3). Es kommt zu wiederkehrenden Auseinandersetzungen zwischen nichtstaatlichen bewaffneten Gruppierungen und den afghanischen nationalen Verteidigungs- und Sicherheitskräften (ANDSF) sowie zwischen den Taliban und neu herangebildeten Splittergruppen des IS (ebd.). Bodenkämpfe verursachen derzeit laut dem Menschenrechtsteam der Hilfsmission der Vereinten Nationen in Afghanistan (UNAMA) weiterhin Rekordzahlen bei den zivilen Opfern (UNAMA 2017: 3). Allein zwischen dem 1. Januar 2017 und dem 30. Juni 2017 gab es 5.243 dokumentierte Fälle, bei denen Zivilist*innen verletzt oder gestorben sind (ebd.) (Abbildung 3.1).

ANZAHL ZIVILER OPFER IN AFGHANISTAN

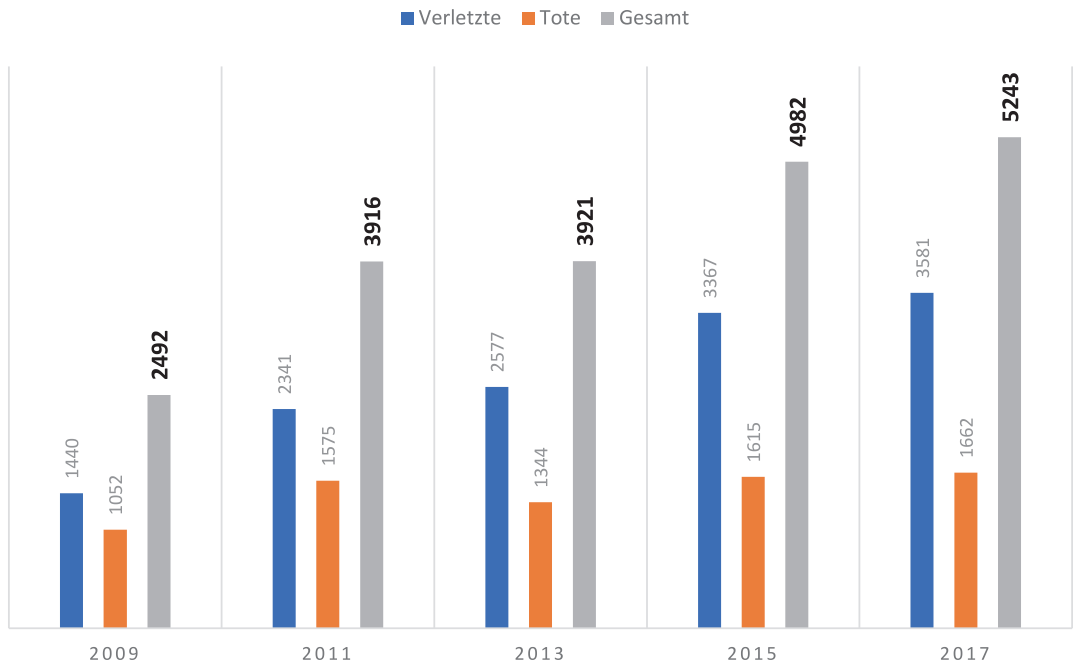

Abbildung 3.1 Anzahl ziviler Opfer in Afghanistan (2009-2017). (Quelle: Eigene Darstellung beruhend auf den Daten des UNAMA-Afghanistan-Mid-Year Reports 2017) 
Ungeachtet dieser Situation unterstellt das BAMF afghanischen Fluchtmigrierten bei der Anhörung sogenannte Flucht- oder Schutzalternativen, die ihre Basis in Art. 8 der EU-Qualifikationsrichtlinie haben. Damit sind innerstaatliche Ausweichmöglichkeiten der Antragsteller*innen gemeint, Orte oder Regionen also, die afghanische Geflüchtete in ihrem Heimatland hätten aufsuchen können, anstatt nach Europa zu flüchten (Lafrai 2013: 177). Nach Art. 8 der EU-Qualifikationsrichtlinie wird das seit den 1980ern entwickelte Konzept der inländischen Fluchtalternative stetig als Rechtfertigung für die Schutzversagung verwendet (ebd.). Art. 8 der EU-Qualifikationsrichtlinie richtet gegenwärtig das Augenmerk vor allem auf mögliche Gebiete, die zum Zeitpunkt der Entscheidung über den Asylantrag als Schutzalternativen gelten. Das bedeutet, interne Schutzzonen werden nicht länger als Alternativen zur Flucht per se, sondern als Alternativen zum internationalen Schutz betrachtet. Daher wird nunmehr statt „Fluchtalternative“ der Begriff „Schutzalternative“ verwendet. Die Richtlinie schreibt deutlich vor, dass sowohl Relevanz als auch Zumutbarkeit der jeweils vorgeschlagenen internen Schutzalternative geprüft werden müssen (UNHCR 2016c: 9). Betreffende Personen müssen demzufolge sicher, legal und ohne weiteres Risiko das Gebiet erreichen können, das von der Bundesbehörde als interne Schutzalternative vorgeschlagen wird. Allgemeine Gegebenheiten und die persönlichen Umstände der Antragsteller*innen müssen laut Art. 8 Abs. 2 EU-Qualifikationsrichtlinie berücksichtigt werden. Laut UNHCR sind mit Blick auf Afghanistan besonders zwei Aspekte zu berücksichtigen (ebd.: 9-10):

„(i) der instabile, wenig vorhersehbare Charakter des bewaffneten Konflikts in Afghanistan hinsichtlich der Schwierigkeit, potenzielle Neuansiedlungsgebiete zu identifizieren, die dauerhaft sicher sind, und

(ii) die konkreten Aussichten auf einen sicheren Zugang zum vorgeschlagenen Neuansiedlungsgebiet unter Berücksichtigung von Risiken im Zusammenhang mit dem landesweit verbreiteten Einsatz von improvisierten Sprengkörpern und Landminen, Angriffen und Kämpfen auf Straßen und von regierungsfeindlichen Kräften auferlegten Einschränkungen der Bewegungsfreiheit von Zivilisten.“

\subsection{Rechtliche Situation}

Nachdem eine Person den Asylantrag in Deutschland gestellt hat, entscheidet das BAMF, ob dem Antrag zugestimmt wird oder nicht. Während des Asylverfahrens wird seitens der Bundesbehörden geprüft, ob Asylbewerber*innen im Falle der Rückkehr in das Herkunftsland gravierende Gefahren drohen. Im Wesentlichen 
gibt es folgende Formen von Schutz: die Anerkennung als Asylberechtigte $(\S 16 \mathrm{a}$ GG) oder als „Flüchtling“ ( $§ 3$ und 4 AsylVfG bzw. GFK ${ }^{4}$ ), subsidiärer Schutz ( $\$ 60$ Abs. 2 bis 4 AufenthG) und humanitär begründete Abschiebungsverbote (§60 Abs. 5 AufenthG). Die Schutzformen haben unterschiedliche Voraussetzungen und unterschiedliche Rechte zur Folge. Der folgende Überblick über diesen Status soll es erlauben, die Verweise aus den Fallstudien korrekt nachzuvollziehen und rechtlich einzuordnen (Tabelle 3.1).

Tabelle 3.1 Unterschiedliche Schutzstatus im Asylprozess. (Quelle: Eigene Darstellung)

\begin{tabular}{l|l}
\hline $\begin{array}{l}\text { Flüchtlingsschutz: } \\
\S \S 3 \text { u. } 4 \text { oder GFK }\end{array}$ & Asylanerkennung \\
\hline $\begin{array}{l}\text { Asylberechtigung: } \\
\text { politische Verfolgung } \S 16 a \text { GG }\end{array}$ & Mittelstarker Schutzstatus \\
\hline $\begin{array}{l}\text { Subsidiärer Schutz: } \\
\S 60 \text { II bis IV AufenthG }\end{array}$ & Schwacher Schutzstatus \\
\hline $\begin{array}{l}\text { Verbot der Abschiebung: } \\
\S 60 \text { Abs. V und VII AufenthG }\end{array}$ & \\
\hline
\end{tabular}

Der Schutzstatus nach Asylanerkennung durch die ersten beiden Gesetze ist weitestgehend identisch. Deutschland gestaltete seit 1949 das Asylrecht mit Art. 16 Abs. 2 GG schrankenlos als Individualrecht aus, was sich erst am 01.07.1993 durch den sogenannten Asylkompromiss änderte. Das subjektive Grundrecht auf Asyl hat zwar prinzipiell nach den Veränderungen weiterhin Bestand, jedoch hat sich die bedingungslose Qualität des Grundrechts stark verändert, weshalb die Änderung von 1993 als eine der einschneidendsten Veränderungen des deutschen Rechts in der Geschichte der BRD verstanden werden kann. So lassen sich der Asylkompromiss und die seit 1993 vorgenommenen Modifikationen als eine restriktive Asylpolitik deuten, die auf die deutliche Verringerung von Asylanträgen in Deutschland abzielt. Der Wortlaut des Art. 16a Abs. 1 GG - „Politisch Verfolgte genießen Asylrecht“ - sichert politisch Verfolgten das Asylrecht zu. Dieses grundlegende und nicht zu begrenzende Recht wird jedoch stark relativiert. Die erste Einschränkung erfolgt durch den zweiten Absatz; laut Abs. 2 darf sich niemand auf das Asylrecht berufen, der aus einem

\footnotetext{
${ }^{4}$ Mit Inkrafttreten des Richtliniengesetzes am 01.12.2013 sind die Genfer Flüchtlingskonvention und das europäische Rechtssystem vollständig ins deutsche Recht implementiert worden. Bis dato wurde durch $§ 60$ I AufenthG darauf verwiesen (Heinhold 2015: 214).
} 
sicheren Drittstaat oder aus einem Mitgliedstaat der Europäischen Gemeinschaft kommt (Hailbronner 1998: 825). Begründet wird dies damit, dass in den Ländern der EU - zumindest de jure - die Anwendung des Abkommens über die Rechtsstellung der Geflüchteten sowie der Konvention zum Schutz der Grundund Menschenrechte gewährleistet ist (Yazicoglu 2000: 11). Falls jemand aus einem sicheren Drittstaat nach Deutschland kommt und in der Bundesrepublik Asyl beantragt, gilt nach Abs. 2 der Anspruch auf Abs. 1 nicht mehr. Dies unterscheidet im Übrigen die internationale von der bundesdeutschen Anerkennung: Gemäß der Genfer Flüchtlingskonvention (GFK) dürfen Geflüchtete auch über Drittstaaten nach Deutschland einreisen. Darüber hinaus können nach Art. 16a Abs. 3 GG bei Personen, die aus sicheren Drittstaaten nach Deutschland kommen, trotz eingelegter Rechtsbehelfe unmittelbar aufenthaltsbeendende Maßnahmen unternommen werden. Seit dem Asylverfahrensbeschleunigungspaket vom 23. Oktober 2015 findet zudem die Abschiebung ohne Ankündigung des genauen Termins statt. Als sichere Drittstaaten versteht der Gesetzgeber primär sämtliche Mitglieder der Europäischen Gemeinschaft, da in diesen die Anwendung der GFK und der Europäischen Menschenrechtskonvention (EMRK) sichergestellt ist. Die Länderliste wurde seit 1993 konsequent erweitert. Im Juni 2016 wurden etwa die maghrebinischen Länder als sichere Herkunftsstaaten festgelegt. Erwähnenswert ist für das Verständnis dieser Einschränkung, dass es sich um einen abstrakten Befund handelt. Personen aus als sicher eingestuften Ländern können demnach im Einzelfall immer noch als politisch Verfolgte anerkannt werden. Kritisch anzumerken ist in diesem Kontext der Umstand, dass die Schutzwirkung der völkerrechtlichen Verträge sich nicht gänzlich mit der des Asylrechts für politisch Verfolgte deckt (Wollenschläger/Schraml 1994: 63). Der Begriff der politischen Verfolgung kann zum Beispiel auch Homosexualität als unabänderliches Merkmal einbeziehen, während die GFK diesen Aspekt nicht explizit herausstellt. Angesichts der fragilen wirtschaftlichen und politischen Lage in vielen Ländern, die formell die GFK und die EMRK ratifiziert haben, besteht darüber hinaus ein begründeter Zweifel, ob die neuen „,sicheren Herkunftsstaaten“ tatsächlich über Strukturen verfügen, die politisch Verfolgten den erforderlichen Schutz bieten können. Das Einreiseverbot und die drohende Zurückweisung von Personen, die aus sicheren Drittstaaten kommen, sind unter anderem aus diesem Grund äußerst kritisch zu bewerten. Wird der antragstellenden Person seitens des BAMF der subsidiäre Schutz gewährt, erhält sie zunächst Aufenthalt für ein Jahr und kann dies mehrfach um zwei Jahre verlängern, wenn im Heimatland die Gefahren fortbestehen, die zur Flucht geführt haben. Der subsidiäre Schutz bietet unbeschränkten Zugang zum Arbeitsmarkt. 
Wurde der Asylantrag einer schutzsuchenden Person abgelehnt, besteht der Flüchtlingsstatus der Duldung. Geduldete haben weder eine legale Berechtigung, sich in Deutschland aufzuhalten, noch eine Arbeitserlaubnis. Betroffene Personen sind prinzipiell ausreisepflichtig. Die rechtlichen Grundlagen zur Duldung sind in $\S 60$ a AufenthG geregelt. Die Abschiebung kann aus menschenrechtlichen bzw. humanitären Gründen, aber auch krankheitsbedingt ausgesetzt werden $(\$ 60 \mathrm{a} 2 \mathrm{c}$ und 2d). Der Krankheitsparagraf spielt für abgelehnte Asylbewerber*innen dieser Studie eine bedeutende Rolle:

,(2c) [...] Der Ausländer muss eine Erkrankung, die die Abschiebung beeinträchtigen kann, durch eine qualifizierte ärztliche Bescheinigung glaubhaft machen. Diese ärztliche Bescheinigung soll insbesondere die tatsächlichen Umstände, auf deren Grundlage eine fachliche Beurteilung erfolgt ist, die Methode der Tatsachenerhebung, die fachlich-medizinische Beurteilung des Krankheitsbildes (Diagnose), den Schweregrad der Erkrankung sowie die Folgen, die sich nach ärztlicher Beurteilung aus der krankheitsbedingten Situation voraussichtlich ergeben, enthalten.

(2d) Der Ausländer ist verpflichtet, der zuständigen Behörde die ärztliche Bescheinigung nach Absatz 2c unverzüglich vorzulegen. Verletzt der Ausländer die Pflicht zur unverzüglichen Vorlage einer solchen ärztlichen Bescheinigung, darf die zuständige Behörde das Vorbringen des Ausländers zu seiner Erkrankung nicht berücksichtigen, es sei denn, der Ausländer war unverschuldet an der Einholung einer solchen Bescheinigung gehindert oder es liegen anderweitig tatsächliche Anhaltspunkte für das Vorliegen einer lebensbedrohlichen oder schwerwiegenden Erkrankung, die sich durch die Abschiebung wesentlich verschlechtern würde, vor. Legt der Ausländer eine Bescheinigung vor und ordnet die Behörde daraufhin eine ärztliche Untersuchung an, ist die Behörde berechtigt, die vorgetragene Erkrankung nicht zu berücksichtigen, wenn der Ausländer der Anordnung ohne zureichenden Grund nicht Folge leistet. Der Ausländer ist auf die Verpflichtungen und auf die Rechtsfolgen einer Verletzung dieser Verpflichtungen nach diesem Absatz hinzuweisen“" (\$ 60a AufenthG).

Zudem muss zwischen zielstaatsbezogenen und inlandsbezogenen Abschiebungshindernissen unterschieden werden (Jentsch 2017: 8). Bei zielstaatsbezogenen Abschiebungshindernissen, wenn also Gefahr oder Rechtsverletzung erst im Heimatstaat bzw. im Zielland der Abschiebung entsteht, ist das BAMF zuständig. Daher sollten Atteste und sämtliche gesundheitsbezogenen relevanten Gesichtspunkte bei der Anhörung vorgetragen werden. Inlandsbezogene Abschiebungshindernisse hingegen meinen die Krankheiten, die sich während des Asylprozesses oder der Abschiebung realisieren (ebd.: 10). Werden die unter $\S 60 \mathrm{a}$ Abs. 2c und 2d AufenthG festgelegten Mindestanforderungen an eine qualifizierte ärztliche Bescheinigung nicht eingehalten, wird das Attest nicht mehr berücksichtigt. 


\subsubsection{Unterbringungsgesetze}

Nach $§ 44$ Abs. 1 Asylgesetz (AsylG) sind die einzelnen Bundesländer dazu verpflichtet,

„für die Unterbringung Asylbegehrender die dazu erforderlichen Aufnahmeeinrichtungen zu schaffen und zu unterhalten sowie entsprechend ihrer Aufnahmequote die im Hinblick auf den monatlichen Zugang Asylbegehrender in den Aufnahmeeinrichtungen notwendige Zahl von Unterbringungsplätzen bereitzustellen“.

Die Versorgung sowie die Unterbringung liegt demnach ausschließlich in den Händen der 16 Bundesländer, wobei die Verweildauer in einer Erstaufnahmeeinrichtung sechs Monate nicht übersteigen soll (Jäger 2016: 5). Der sogenannte Königsteiner Schlüssel ${ }^{5}$ regelt hierbei die Aufnahmequoten einzelner Länder. Geflüchtete sind in diesem Kontext reine Objekte der Verwaltung. In Bezug auf die Aufnahme, Zuweisung und Unterbringungsform haben sie keinerlei Mitspracherecht. Größe, Beschaffenheit oder Ausstattung der Erstaufnahmeeinrichtung sind im AsylG nicht festgelegt (Renner 2005: 1023). Lediglich die Einheit der Familie muss nach § 46 Abs. 3 Satz 2 des AsylG gewährleistet werden. Die Bevormundung und Kontrolle hinsichtlich der Unterbringung endet erst mit der Anerkennung als asylberechtigte Person. Im Falle einer rechtskräftigen Ablehnung des Asylantrags und der Aussetzung der Abschiebung mit einer Duldung nach § 60a AufenthG kann die Ausländerbehörde anordnen, dass weiterhin die Wohnauflage gemäß § 51 Abs. 6 AufenthG gilt (Wendel 2014: 11). Dies ist ein entscheidender Punkt für die vorliegende Studie, da die Interviewpartner*innen von diesem Gesetz betroffen sind. Im Stadtstaat Berlin regelt das Landesamt, das unmittelbar der Senatsverwaltung für Gesundheit und Soziales untersteht, die Akquisition, die Errichtung, den Betrieb, die Belegung und die Schließung von Aufnahmeeinrichtungen und Gemeinschaftsunterkünften für Asylbewerber*innen. Die Unterbringung in einer Erstaufnahmeeinrichtung (EAE) erfolgt nach $\S 47$ AsylG für diejenigen, die den Asylantrag noch nicht gestellt haben, und in Gemeinschaftsunterkünften nach $\S 53$ AsylG für diejenigen, die ihren Asylantrag bereits gestellt haben. Für beide Unterkunftstypen schließt das Land Berlin mit Betreibern Verträge ab. Bundesweite Mindeststandards für die Unterbringung von Asylbewerber*innen existieren nicht, da die Unterbringung der kommunalen Selbstverwaltung unterliegt. Allerdings gibt es in einigen Bundesländern verbindliche Mindeststandards, in anderen hingegen nur Empfehlungen (Tabelle 3.2).

\footnotetext{
${ }^{5}$ Der Königsteiner Schlüssel regelt die Verteilung der in Deutschland ankommenden Fluchtmigrierten; er berücksichtigt die Bevölkerungszahl und die Steuereinnahmen in jedem Bundesland und bestimmt danach die aufzunehmende Zahl an Geflüchteten in Prozent.
} 
Tabelle 3.2 Mindeststandards für Flüchtlingsunterkünfte. (Quelle: Wendel 2014: 35-36. Eigene Darstellung)

\begin{tabular}{l|l}
\hline Bundesland & Regelung der Mindeststandards \\
\hline Rheinland-Pfalz & Keine Mindeststandards \\
\hline Saarland & Keine Mindeststandards \\
\hline Nordrhein-Westfalen & Keine Mindeststandards \\
\hline Niedersachsen & Keine Mindeststandards \\
\hline Hessen & Keine Mindeststandards \\
\hline Hamburg & Keine Mindeststandards \\
\hline Bremen & Keine Mindeststandards \\
\hline Bayern & Empfehlungen für Mindeststandards \\
\hline Sachsen & Empfehlungen für Mindeststandards \\
\hline Sachsen-Anhalt & Empfehlungen für Mindeststandards \\
\hline Schleswig-Holstein & Empfehlungen für Mindeststandards \\
\hline Thüringen & Verbindliche Mindeststandards \\
\hline Mecklenburg-Vorpommern & Verbindliche Mindeststandards \\
\hline Berlin & Verbindliche Mindeststandards \\
\hline Brandenburg & Verbindliche Mindeststandards \\
\hline
\end{tabular}

Das Fehlen von Mindeststandards deutet nicht zwangsläufig auf eine menschenunwürdige Unterbringung von Asylbewerber*innen hin. Viele Städte haben sich aus freien Stücken zu Mindeststandards verpflichtet, etwa Köln, Hannover und Osnabrück (Wendel 2014: 37). Das Land Hessen beispielsweise hat - ohne gesetzliche Verpflichtung - die von Flüchtlingsräten und Wohlfahrtsverbänden seit langem geforderte Mindestwohn- und Schlaffläche pro Person durchgesetzt. Die Anzahl der untergebrachten Personen pro Raum und die Größe der Unterkunft bilden jedoch zentrale Punkte, die gemäß den Forderungen von Flüchtlingsräten und Wohlfahrtsverbänden bundesweit festgelegt werden müssen. Blinde Flecken der Mindeststandards sind zum einen das Fehlen eines Verbots von Wohncontainern und zum anderen das Ignorieren von Standards bezüglich der Wohnqualität, wie der Zugang zu Sprachmittler*innen, Sozialarbeiter*innen und zur Heimleitung (ebd.). In Berlin zeigt sich seit 2015 die Situation, dass die Sollvorschrift hinsichtlich der Mindestwohnfläche pro Person nicht immer berücksichtigt wird. Laut Qualitätsanforderungen des Landesamtes Berlin müssen Einzelzimmer mindestens $9 \mathrm{~m}^{2}$ groß sein; für jede zusätzliche Person müssen 
mindesten $6 \mathrm{~m}^{2}$ und für jedes Kind bis zu sechs Jahren mindestens $4 \mathrm{~m}^{2}$ reiner Wohnfläche zur Verfügung stehen (Landesamt Berlin 2017). Laut Drucksache des deutschen Bundestages stieg Ende 2016 die Anzahl geduldeter Personen auf 725.000 an (BT-Drs. 19/3860 (2018): 2). 2017 lebten 556.449 abgelehnte Asylbewerber*innen (davon 166.000 Geduldete) in der Bundesrepublik (BT-Drs. 19/633 (2018): 2). Besonders in Ballungsgebieten wie Berlin, die für unterschiedliche Personengruppen attraktiv sind, kommt es u. a. deshalb zu verheerenden Wohnraumproblemen. Bei nur 125.000 Sozialwohnungen $^{6}$ wird in Berlin seit drei Jahrzehnten vergeblich darum gekämpft, die Anzahl der Sozialwohnungen zu vermehren. Infolge der fehlgeschlagenen Sozialpolitik des Stadtstaates und des plötzlichen Anstiegs der Asylbewerber*innenzahlen bildet der Wohnraum eine zentrale Dimension, an der sich viele soziale Verwicklungen erkennen lassen, die direkt oder indirekt für soziale Ungleichheit verantwortlich sind. Geduldete Personen und all diejenigen, die auf die Bearbeitung ihres Asylprozesses warten, sind in der Regel im Unterschied zu anerkannten Asylbewerber*innen, die einen Schutzstatus erhalten haben, verpflichtet, in einer Aufnahmeeinrichtung zu bleiben. Gemäß Asylbewerberleistungsgesetz (AsylbLG) ${ }^{7}$, das Leistungen zur Grundsicherung an Ausländer*innen, die sich als Asylsuchende, mit Duldung oder mit bestimmten Aufenthaltserlaubnissen in Deutschland aufhalten, dürfen sie zudem weder arbeiten noch ihre eigenen Mahlzeiten zubereiten.

\subsubsection{Asylverfahren}

Nachdem asylsuchende Personen Deutschland erreicht haben, müssen sie sich registrieren lassen und werden anschließend in eine Aufnahmeeinrichtung gebracht. Die EAE soll laut $\S 47$ AsylG bis zu sechs Wochen, längstens jedoch bis zu sechs Monaten von Asylbewerber*innen bewohnt werden. Allerdings kamen 2015 über 55.000 Asylbewerber*innen nach Berlin, so dass solche Verordnungen und Regelungen nicht mehr einzuhalten waren. Nachdem sich neu ankommende Geflüchtete beim Landesamt als Asylbewerber*innen registriert haben, wird auf der Grundlage des Königsteiner Schlüssels entschieden, in welche Stadt die antragstellende Person transferiert werden soll. Berlin muss demnach über 5 Prozent der Asylbewerber*innen bundesweit aufnehmen und unterbringen. Die EAEs sollen geflüchteten Personen zunächst Obdach und

\footnotetext{
${ }^{6}$ Senat für Stadtentwicklung Berlin, Wohnungsarten und Wohnungsanmietung.

${ }^{7}$ Das AsylbLG verdrängt in seinem Anwendungsbereich das SGB II und XII und ist erstmalig am 01.10.1993 in Kraft getreten.
} 
lebensnotwendige Dinge bieten, damit möglichst rasch eine Sozialwohnung bezogen werden kann. Dies war bereits vor 2015 unrealistisch und gestaltete sich seit dem Sommer 2015 für unzählige Asylbewerber*innen als unmöglich. Neben dem überfüllten Berliner Wohnungsmarkt verhindern Mietobergrenzen seitens des Sozialamtes $^{8}$ und die Sprachbarriere der Asylantragsteller*innen es, angemessene Unterkünfte zu finden. Währenddessen werden die meisten Asylbewerber*innen Berlins in 131 Unterkünften (74 davon Notunterkünfte) untergebracht. Zwischen Einreise und Entscheidung des BAMF vergeht durchschnittlich ein Jahr. Doch dies regelt die Bundesbehörde nach eigenen Maßstäben von Herkunftsland zu Herkunftsland anders: Somalische Asylsuchende warten derzeit rund zweieinhalb Jahre, bis sie die Entscheidung der Anhörung erhalten, syrische Antragsteller*innen hingegen nur dreieinhalb Monate (BT-Drs. 18/11262) (Abbildung 3.2).

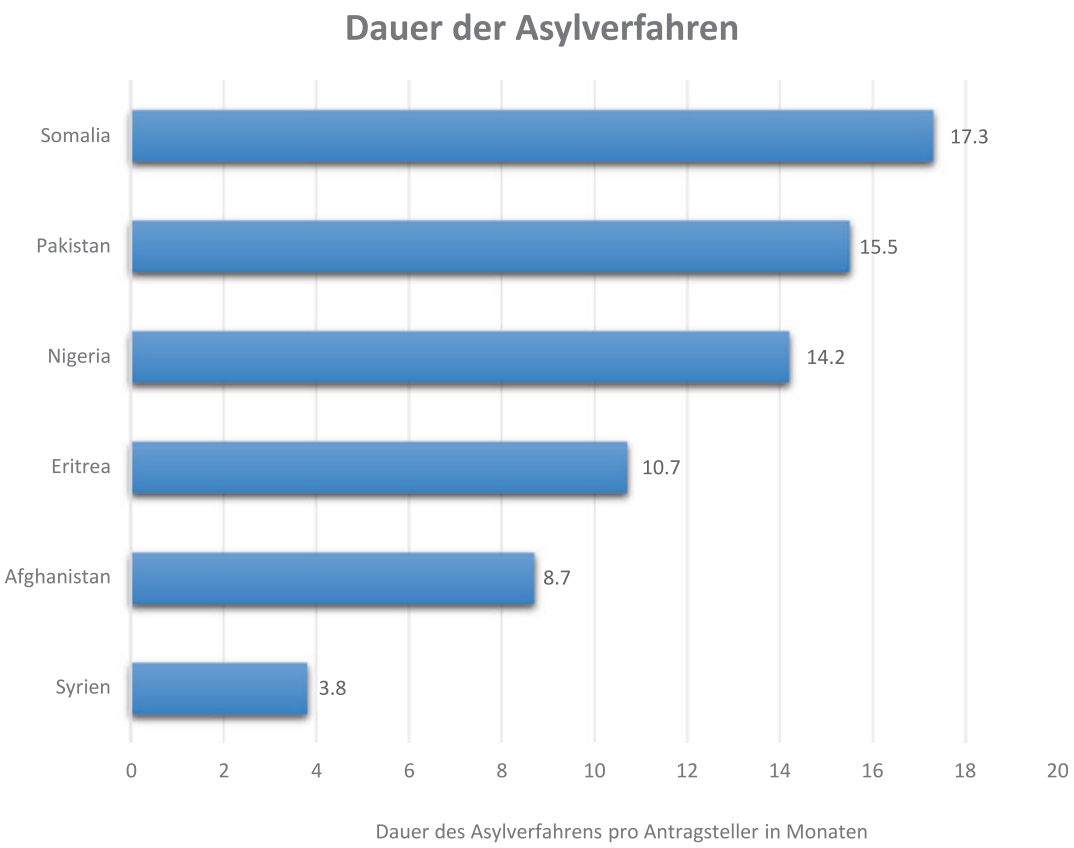

Abbildung 3.2 Dauer der Asylverfahren 2016 (in Monaten). (Quelle: Eigene Darstellung beruhend auf den Daten der Drucksache des deutschen Bundestages (BT-Drs. 18/11262))

${ }^{8}$ Geflüchtete bekommen in diesem Zeitraum dieselben Bezüge wie Arbeitslosengeld-IIEmpfänger. 
Rund 25 Prozent aller Asylanträge im Jahr $2016^{9}$ wurden abgelehnt, was eine Schutzquote von 71,4 Prozent ergibt, wenn man die ungeprüften Fälle ${ }^{10}$ abzieht. Hinzu kommt, dass der Großteil der BAMF-Entscheide von Asylbewerber*innen angefochten und vor dem Verwaltungsgericht ausgehandelt wird. Dies dauert wiederum etwa vier Jahre, so dass abgelehnte Asylbewerber*innen faktisch etwa fünf Jahre in Notunterkünften und EAEs ausharren müssen. Die schwierige Wohnungssituation in Großstädten wie Berlin kommt erschwerend hinzu (Abbildung 3.3).

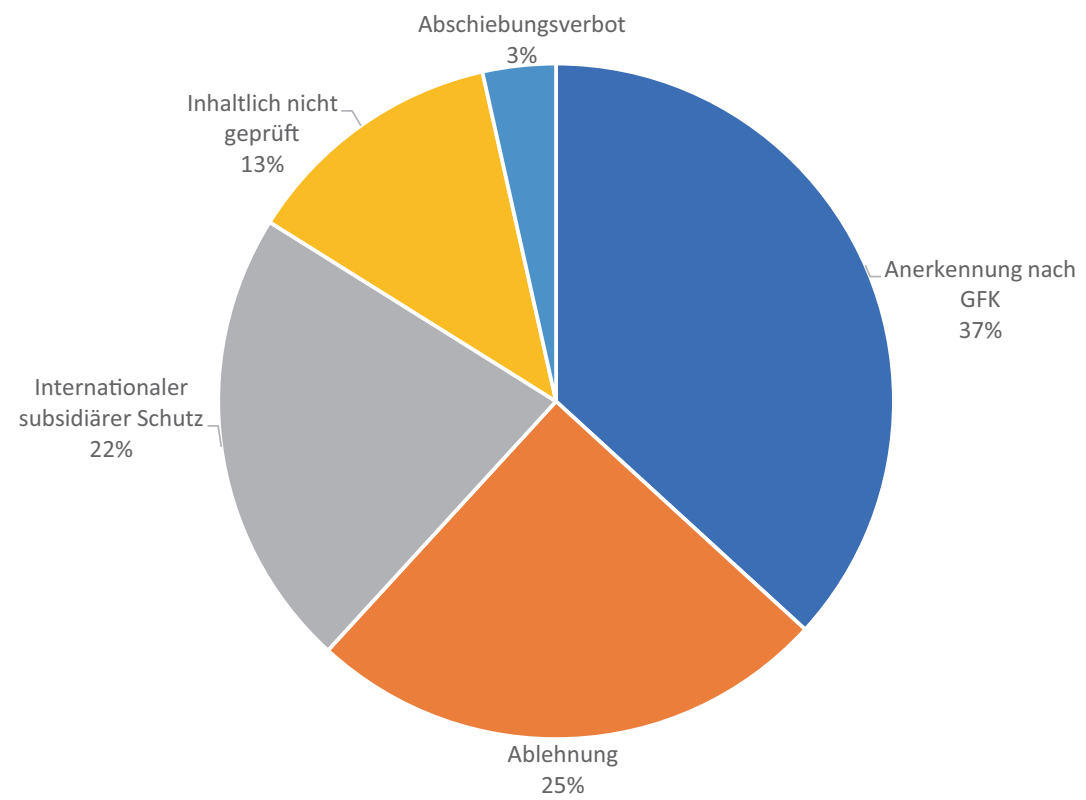

Abbildung 3.3 BAMF-Entscheidungen 2016 (in Prozent). (Quelle: Eigene Darstellung beruhend auf den Daten des BAMF-Asylgeschäftsberichts von 2017)

Eine Schutzquote von über 70 Prozent ist eine beeindruckende Zahl, doch die damit einhergehende sinkende Schutzqualität sowie die repressiv gewordene Entscheidungspraxis dürfen nicht ausgeklammert werden. Tatsächlich ist dies

${ }^{9}$ Im Jahr 2016 registrierte das BAMF 745.545 Erst- und Folgeanträge und nahm 116.616 Asylanträge entgegen (BAMF, Asylgeschäftsbericht).

${ }^{10}$ Der Großteil nicht geprüfter Fälle besteht aus Dublin-Verfahren und Folgeanträgen. 
ein enormes Problem für Menschen, die künftig in Deutschland Asyl beantragen möchten. Es geht also nicht um Abschiebungen, wie öffentliche Debatten und Medienberichterstattungen zuweilen vermuten lassen, sondern vielmehr um die Frage, wie mit abgelehnten Asylbewerber*innen umgegangen werden soll. Die Anzahl der Abschiebungen ist relativ gering, 2016 waren es gerade einmal 324 afghanische Asylbewerber*innen bei insgesamt 25375 Abschiebungen; 2019 sind es 931 Afghan*innen bei insgesamt 22097 Abschiebungen(BT-Drs. 19/18201). Die gesellschaftlichen Konsequenzen müssen mit Blick auf die steigenden Zahlen geduldeter Personen in Deutschland gründlich bedacht werden. Im Vorjahr 2015 lag die Schutzquote noch bei 60,4 Prozent, doch beschied das BAMF weitaus weniger Ablehnungen bzw. Duldungen (Abbildung 3.4).

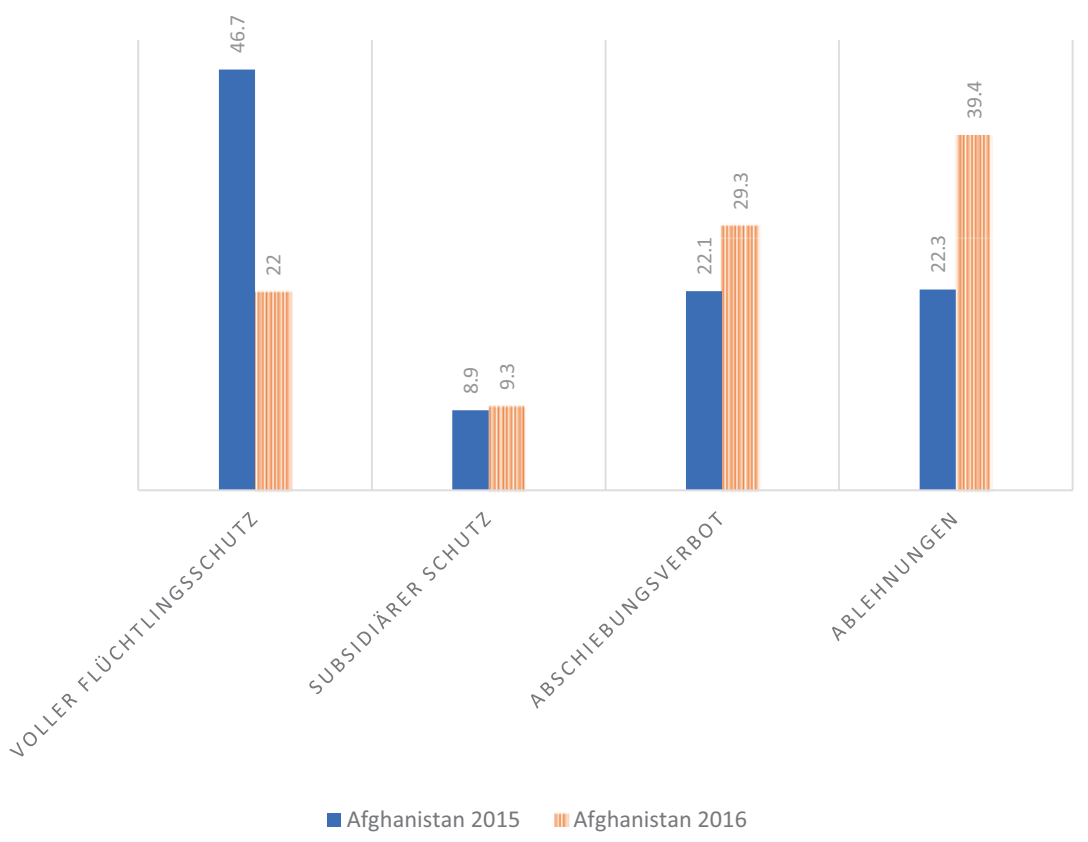

Abbildung 3.4 Schutzqualität bei BAMF-Entscheidungen 2015-2016 (in Prozent). (Quelle: Eigene Darstellung beruhend auf den Daten des BAMF-Asylgeschäftsbericht (2017))

Besonders für Afghanistan ist diese Abwärtsentwicklung der Schutzqualität von einer Anerkennungsquote von 55,8 Prozent (2016) auf 44,1 Prozent (erstes Halbjahr 2017) deutlich zu spüren. Gleichzeitig steigt die Zahl ankommender afghanischer Geflüchteter, wie Abbildung 3.5 zeigt. 
Abbildung 3.5 Anstieg

afghanischer

Asylbewerber*innen in

Deutschland. (Quelle:

Eigene Darstellung

beruhend auf den

Daten der BAMF-

Asylgeschäftsbericht von

2017)
ANZAHL ANKOMMENDER

GEFLÜCHTETER AUS

AFGHANISTAN
300,000

250,000

200,000

150,000

100,000

50,000

0

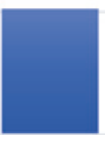

2010

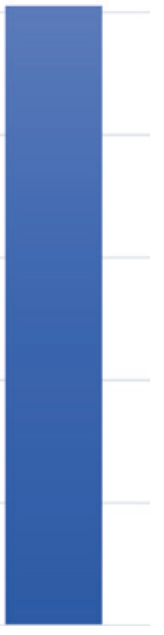

2016

Erstaunlicherweise reagiert die Bundesregierung auf die ungewöhnlichen Fluchtbewegungen mit der plötzlichen Verschärfung der Anforderungen für Schutzbestimmungen, obwohl sich die Sicherheitslage in Afghanistan etwa durch das Hinzukommen des Islamischen Staates (IS) wesentlich verschlimmert hat. Es ist festzuhalten, dass die steigende Zahl der Asylanträge die politische Forderung nach asyl- und aufenthaltsrechtlichen Verschärfungen aufkommen lässt. Die Große Koalition aus CDU/CSU und SPD brachte mit den drei sogenannten „Asylpaketen“ seit Oktober 2015 die härtesten Verschärfungen des Asylrechts der letzten Dekaden auf den Weg. Zu nennen sind in diesem Kontext die Ausweitung der ,sicheren Herkunftsstaaten“, die Einführung „beschleunigter Asylverfahren" sowie die menschenrechtlich kontroverse Residenzpflicht für Asylbewerber*innen. All dies trägt entscheidend zu einer grundsätzlichen Kategorisierung und (ethnischen) Hierarchisierung (siehe Fallstudie Nr.1) der Geflüchteten bei. 


\subsection{Zum Begriff „Flüchtlingskrise“}

In der vorliegenden Studie wird der Begriff „Flüchtlingskrise“ so verstanden, dass er auf zwei krisenhafte Momente verweist, die untrennbar mit „dem Flüchtling" des 21. Jahrhunderts verbunden sind: zum einen auf die Krise des EU-Grenzregimes und zum anderen auf die Krise der „Figur des Flüchtlings“, dessen Rechte aufgrund weltweit veränderter Bedingungen nicht mehr gewahrt werden. Die Unzulänglichkeiten der EU-Flüchtlingspolitik verdichteten sich im Sommer 2015 aufgrund unterschiedlicher Faktoren zu einer krisenhaften Situation. Der Streit zwischen den europäischen Ländern stellte eine untragbare Situation für zahllose schutzsuchende Menschen dar (Wessel 2017). Über 30 Jahre lang versuchten wenige westliche Wohlstandsnationen ein höchst selektives, mehrstufiges Grenzregime zu etablieren, das sich durch eine rigide Abschottung von Geflüchteten bei gleichzeitiger Binnenfreizügigkeit auszeichnet. Migration und Flucht wurden damit zum Gegenstand von Überwachung und das Mittelmeer zu einer militarisierten Todeszone (Hess et al. 2017: 6). Der Zerfall nordafrikanischer Pufferstaaten, der Kollaps des Dublin-Systems sowie die Öffnung Deutschlands am 5. September 2015 sorgten für unkontrollierte Flucht- und Migrationsbewegungen nach Europa (Luft 2016: 7). Bis zum März 2016 erreichten innerhalb eines Jahres über eine Million Geflüchtete die EU, doch seitdem versucht die EU das Grenzregime in deutlich verschärfter Form wiederherzustellen (Hess et al. 2017: 11). Die sich zuspitzende Situation an den Außengrenzen Europas sowie Bilder von Stacheldrähten, Bootsflüchtlingen, überfüllten „Flüchtlingscamps“, Militär, Polizei und NGOs werden mit dem Begriff „Flüchtlingskrise“ assoziiert (Wessel 2017). Anders ausgedrückt ist das etablierte Grenzregime vollständig kollabiert (Hess et al. 2017: 7). Dies ist eine ausschlaggebende Seite der „Flüchtlingskrise“. Durch die Notstandsrhetorik von Politiker*innen und dem Großteil der Medien erscheint der Zusammenbruch des Grenzregimes jedoch als ein ,unglücklicher Unfall“ (ebd.: 8).

Eine weitere Facette der sogenannten „Flüchtlingskrise“ deutet auf die Krise der Flüchtlingsfigur hin, denn diese durchläuft eine grundlegende Transformation (Wessel 2017). Die einschlägigen Fluchtursachen, also Kriege, militärische Auseinandersetzungen, humanitäre Katastrophen, Armut und Hunger, fehlender Schutz von Menschenrechten, die steigenden Auswirkungen der Klimaveränderung, und der daraus folgende Anstieg der Fluchtbewegungen und der Flüchtlingszahlen bringen eine Flüchtlingsfigur hervor, die sich erheblich von jener unterscheidet, wie sie das internationale Recht anhand der Flüchtlingseigenschaften umrissen hat. Der größte Teil Fluchtmigrierender weltweit sind laut internationalem Flüchtlingsrecht keine „Flüchtlinge“, weil sie nicht vor 
starken Staaten fliehen, die sie unterdrücken (ebd.). Sie fliehen aus schwachen, zerfallenden Staaten, die nicht in der Lage sind, eine systematische Verfolgung zu betreiben, aber eben auch nicht, vor Gefahren zu schützen. Die Definition des Flüchtlingsbegriffs ${ }^{11}$ laut GFK berücksichtigt somit nicht die wesentlichen Fluchtursachen wie etwa Bürgerkrieg (Syrien), kriegerische Auseinandersetzungen (Afghanistan, Somalia), politische und religiöse Verfolgung (Myanmar, Palästina) und politische Instabilität (Eritrea, Sudan, Libyen) (Grönheim 2019: 20). Dabei ist $\mathrm{zu}$ beachten, dass die allermeisten Geflüchteten auf der Erde internally displaced persons (Binnenvertriebene) sind, weil sie sich die kostspielige Flucht ins Ausland nicht leisten können ${ }^{12}$. Die Hauptaufnahmeländer liegen aus demselben Grund außerhalb der Wohlstandsnationen des Westens. Bis 2014 befand sich kein einziger EU-Staat unter den zehn Ländern ${ }^{13}$, die weltweit am meisten geflüchtete Personen aufnehmen. Flucht und Asyl ereignen

${ }^{11}$ Im Sinne dieses Abkommens findet der Ausdruck ,Flüchtling“ auf jede Person Anwendung,

1. die in Anwendung der Vereinbarungen vom 12. Mai 1926 und 30. Juni 1928 oder in Anwendung der Abkommen vom 28. Oktober 1933 und 10. Februar 1938 und des Protokolls vom 14. September 1939 oder in Anwendung der Verfassung der Internationalen Flüchtlingsorganisation als Flüchtling gilt. Die von der internationalen Flüchtlingsorganisation während der Dauer ihrer Tätigkeit getroffenen Entscheidungen darüber, dass jemand nicht als Flüchtling im Sinne ihres Statuts anzusehen ist, stehen dem Umstand nicht entgegen, dass die Flüchtlingseigenschaft Personen zuerkannt wird, die die Voraussetzungen der Ziffer 2 dieses Artikels erfüllen;

2. die infolge von Ereignissen, die vor dem 1. Januar 1951 eingetreten sind, und aus der begründeten Furcht vor Verfolgung wegen ihrer Rasse, Religion, Nationalität, Zugehörigkeit zu einer bestimmten sozialen Gruppe oder wegen ihrer politischen Überzeugung sich außerhalb des Landes befindet, dessen Staatsangehörigkeit sie besitzt, und den Schutz dieses Landes nicht in Anspruch nehmen kann oder wegen dieser Befürchtungen nicht in Anspruch nehmen will; oder die sich als staatenlose infolge solcher Ereignisse außerhalb des Landes befindet, in welchem sie ihren gewöhnlichen Aufenthalt hatte, und nicht dorthin zurückkehren kann oder wegen der erwähnten Befürchtungen nicht dorthin zurückkehren will. Für den Fall, dass eine Person mehr als eine Staatsangehörigkeit hat, bezieht sich der Ausdruck ,das Land, dessen Staatsangehörigkeit sie besitzt“, auf jedes der Länder, dessen Staatsangehörigkeit diese Person hat. Als des Schutzes des Landes, dessen Staatsangehörigkeit sie hat, beraubt, gilt nicht eine Person, die ohne einen stichhaltigen, auf eine begründete Befürchtung gestützten Grund den Schutz eines der Länder nicht in Anspruch genommen hat, deren Staatsangehörigkeit sie besitzt (GFK Art. 1 A(1) und (2)).

${ }^{12}$ Von den 80 Millionen Geflüchteten waren 2020 etwa 45,7 Millionen Binnenvertriebene (UNO 2020). Allein in Afghanistan sind seit der statistischen Erhebung 2012 des UN-Nothilfebüros Ocha über 2 Millionen Binnenvertriebene gezählt worden (OCHA 2019).

${ }^{13}$ Die zehn aufnahmestärksten Länder waren 2014/15 die Türkei, Pakistan, Libanon, der Iran, Äthiopien, Jordanien, Kenia, Tschad, Uganda, China (UNHCR 2014: 12). 
sich in der Regel in der direkten Nachbarschaft von Krisenregionen (Hoesch 2018: 134). Die eigentliche „Krise“ spielt sich insofern nicht innerhalb der EUStaaten ab, sondern im globalen Süden. Ein weiterer Aspekt der „Krise“ besteht in der veränderten Situation Vertriebener und Geflüchteter weltweit. „Der Flüchtling“ und „die Flüchtlingskrise“ werden als Termini verstanden, die Fluchtmigrierende fremdbestimmen und das binäre System eurozentrischer Denkweisen (siehe Abschn. 4.3) begünstigen. Diese Begrifflichkeiten produzieren langfristig einen rassistischen Diskurs, der im europäischen Grenzregime ,ungewollte Flüchtlinge" von gewünschten „hochqualifizierten Migrant*innen" (siehe Abschn. 4.3) unterscheidet. Durch die unterschiedliche Implementierung des Art. 1 A(2) GFK wird zudem eine ungenaue Identität „des Flüchtlings“ geschaffen, anstatt eine allgemeingültige und angemessene Definition Fluchtmigrierender $\mathrm{zu}$ entwickeln, die den skizzierten gegenwärtigen politischen Konstellationen Rechnung trägt.

Open Access Dieses Kapitel wird unter der Creative Commons Namensnennung 4.0 International Lizenz (http://creativecommons.org/licenses/by/4.0/deed.de) veröffentlicht, welche die Nutzung, Vervielfältigung, Bearbeitung, Verbreitung und Wiedergabe in jeglichem Medium und Format erlaubt, sofern Sie den/die ursprünglichen Autor(en) und die Quelle ordnungsgemäß nennen, einen Link zur Creative Commons Lizenz beifügen und angeben, ob Änderungen vorgenommen wurden.

Die in diesem Kapitel enthaltenen Bilder und sonstiges Drittmaterial unterliegen ebenfalls der genannten Creative Commons Lizenz, sofern sich aus der Abbildungslegende nichts anderes ergibt. Sofern das betreffende Material nicht unter der genannten Creative Commons Lizenz steht und die betreffende Handlung nicht nach gesetzlichen Vorschriften erlaubt ist, ist für die oben aufgeführten Weiterverwendungen des Materials die Einwilligung des jeweiligen Rechteinhabers einzuholen.

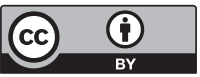

